\title{
Braquiterapia en el cáncer de próstata localizado
}

\author{
Abascal Junquera JM*,Hevia Suarez M, *Abascal García JM*, Abascal García R*, \\ Gonzalez Suárez H**, Alonso A**, Juan Rijo G**, Prada PJ**. \\ *Servicio Urología. **Servicio Radioterapia. Hospital Universitario Central de Asturias.
}

Actas Urol Esp. 2007;31(6):617-626

\section{RESUMEN}

BRAQUITERAPIA EN EL CÁNCER DE PRÓSTATA LOCALIZADO

Introducción y objetivos: Teniendo en cuenta la alta frecuencia del cáncer de próstata en estadios localizados, existen actualmente técnicas mínimamente invasivas que compiten con la cirugía clásica. Una de ellas es la Braquiterapia de Baja Tasa de Dosis con implantes permanentes de semillas de Yodo- 125.

El objetivo del presente trabajo es exponer nuestra experiencia desde el año 1998 en que realizamos el primer tratamiento hasta el día de hoy. Se analizan los resultados y la morbilidad de los pacientes con un seguimiento de 7 años y medio.

Material y Métodos: Un total de 800 pacientes fueron tratados con Baja Tasa de Dosis, con edad media de 68 años y rango entre 48 y 83 años. En todos los pacientes las semillas de I125 fueron utilizadas con Rapid-Strand, con técnica de carga periférica y mediante planificación intraoperatoria.

Resultados: La tasa de complicaciones urinarias fueron del 3\% de RAO, y del 0,2\% de incontinencia urinaria. La morbilidad sobre el aparato digestivo fue de un $12 \%$ de sangrados intermitentes, $2 \%$ de proctitis, y un $0,3 \%$ de fistulas rectales.

Palabras clave: Braquiterapia. Cáncer de próstata localizado.

\section{ABSTRACT \\ BRACHYTERAPY IN THE LOCALIZED PROSTATE CANCER}

Introduction and objectives: Considering the high frequency of localized prostate cancer in stages, at the moment there are minimally invasive techniques that compete with the classic surgery. One of them is the Low Dose Rate (LDR) Brachytherapy with permanent implants of I125 seeds.

The objective of the present study is to expose our experience from the year 1998, when we made the first treatment, until today. The results and the morbidity of the patients over a 7 and a half years period are analyzed.

Material and methods: A total of 800 patients were treated with LDR brachytherapy, with average age of 68 years and range between 48 and 83 years. In all patients the I125 seeds were used with Rapid-Strand and peripheral load by means of intraoperative planning.

Results: The urinary rate of complications was of $3 \%$ of AUR, and $0.2 \%$ of urinary incontinence. The morbidity on the digestive apparatus was of a $12 \%$ intermittent bleeding, $2 \%$ of proctitis, and a $0.3 \%$ of rectal fistulas.

Keywords: Brachytherapy. Localized prostate cancer.

$\mathrm{E}$ s conocido que el carcinoma de próstata es la segunda causa de muerte por cáncer en el varón, y que su incidencia está aumentando, ya sea por el envejecimiento de la población, la mayor precisión en el despistaje o el aumento del número de biopsias sistemáticas de próstata.
El resultado de todos estos factores es un gran incremento del diagnóstico de cáncer localizado en la glándula, y por lo tanto susceptible de un tratamiento curativo, y que además cada vez se ve en gente más joven.

Las posibilidades de tratamiento en estos casos seleccionados van desde la observación y 
vigilancia hasta la prostatectomía radical, con sus variantes de cirugía laparoscópica, con o sin robot, y las técnicas menos invasivas como la braquiterapia, radioterapia conformada o crioterapia.

Las pequeñas variaciones de resultados a largo plazo independientemente de la técnica utilizada y la menor morbilidad de estas últimas fue lo que impulsó su desarrollo, y las introdujo en el abanico de posibilidades terapéuticas en el tratamiento del carcinoma de próstata localizado ${ }^{1}$.

Las técnicas cada día más evolucionadas, tienden a minimizar la agresión quirúrgica, equiparando, a menudo que el carcinoma es más localizado y mejor diferenciado, la supervivencia a la de la prostatectomía radical abierta, laparoscópica.

La evolución técnica parte de los axiomas antiguos, mejorando la incisión, sangrado, tiempo de ingreso, y complicaciones. Así la cirugía abierta se ve igualada en resultados por la Laparoscópica, Laparoscópica robótica y mejorada en los aspectos que antes comentábamos.

También en la radioterapia ocurre de forma similar, y desde inicios del siglo pasado, Young 1910, Pasteau $1914^{2}$ utilizan radio por vía intrauretral, posteriormente Barringer ${ }^{3}$ lo aplica por vía transperineal; Flocks por vía retropúbica inicia el tratamiento mediante Au coloidal, que posteriormente y con I125 llevaría acabo Whitmore $^{4}$ en Nueva York.

El primero que utilizó la vía transperineal para implante de semillas radiactivas fue Hans Holm en la Universidad de Copenhague ${ }^{5}$ en 1980, y Blasko y Haakon Radge en el Northwest Hospital de Seattle $^{6}$ los que por último normalizaron la utilización de rejilla graduada, y programas computarizados que permitieron la reproducción del método, y una distribución uniforme y controlada de la radiación.

La palabra braqui viene del griego "brachys", que significa corto, de ahí que la braquiterapia sea el tratamiento mediante fuentes radiaoactivas encapsuladas aplicadas a corta distancia del tejido a tratar.

Nos vamos a referir a la braquiterapia intersticial que es aquella en la que se utilizan unas agujas huecas para aplicar en el interior de los tejidos. Hay dos tipos de braquiterapia intersti- cial una con implante temporal y otra con implante permanente. En la temporal colocamos las agujas de forma dirigida por ecografía, vía transperineal, en la próstata; las agujas son huecas y la dosis de irradiación se administra de forma única, en pocos minutos y a través de una máquina de carga diferida automática.

$\mathrm{El}$ elemento radiactivo que proporciona dicha irradiación es el iridio 192 (alta tasa de dosis). Una vez finalizado el tratamiento se retira el implante, se repite el tratamiento a los 15 días, y posteriormente se complementa con radioterapia externa. (Protocolo de HDR en Fase II).

En el caso del implante permanente de Baja Tasa de dosis se aplica la misma técnica, pero usando unas semillas radiactivas de 1125 o Paladio103, que quedan en el interior de la próstata, y se distribuyen uniformemente para lograr una irradiación completa de la glándula.

El primer implante que se practicó en nuestro país, de tipo intersticial con Au198, abordando la glándula por vía retropúbica (Pfannestield), se realizó en el Hospital General de Asturias en 1981

Se trató por aquel entonces una veintena de pacientes, complementados posteriormente con radioterapia externa. La técnica se abandonó por la necesidad de anestesia, abordaje quirúrgico, la colocación a mano alzada de las semillas, elevada morbilidad y como consecuencia de esto, la irregular distribución de los implantes y una dosificación descontrolada.

El desarrollo de las técnicas de imagen, sobre todo de la ecografía con transductor rectal, y la aplicación de programas de medición y de planificación de la dosimetría, le dan un gran impulso a la técnica.

En 1983, como ya habíamos mencionado, Holm, fue el primero en utilizar la vía perineal para implante de semillas, guiado por ecografía transrectal. Posteriormente en 1984, Blasko y Radge en Seattle fueron los grandes impulsores de esta técnica, con I125, incorporándose el Paladio en 1986, que hoy en día ya apenas se utiliza.

Por otro lado en 1987 en Alemania se lleva a cabo la primera aplicación de Alta Tasa (HDR), que sistematizó y divulgó en EEUU Álvaro Martines, quien tuteló el primer implante de Alta Tasa en España, que realizamos en el Hospital General de Asturias en 1998. 
El primer tratamiento con semillas de I125, se realizó por primera vez en España en 1999, en el Hospital General de Asturias. Desde aquella fecha venimos simultaneando ambas técnicas de Alta y Baja tasa para el tratamiento del cáncer de próstata localizado.

A 1 de Marzo de 2007, se trataron en Asturias 800 pacientes con I125, y 330 HDR, con Iridio.

La braquiterapia, teniendo en cuenta que el cáncer de próstata es dosis dependiente, permite aplicar mayores dosis de irradiación, hasta 14.500 cGy con Baja Tasa como dosis única, y se consigue una dosis equiparable biológica aproximada de 15.500 cuando se asocia a radiación externa.

Con la Alta Tasa se pueden alcanzar dosis biológicas de 12.000 cGy a 13.000 cGy, mientras que con la radioterapia externa no se pueden superar los 7.500 cGy, y con conformada la dosis máxima es también de $8.000 c_{G y}^{9,10}$.

Tiene además como ventajas que la caída de la dosis fuera de la glándula es rápida, con lo que los tejidos periprostáticos no sufren o en muy escasa medida irradiación, y al estar fija por las agujas en el momento del implante no está influenciada por movimientos de la glándula. Además es un tratamiento ambulatorio, mínimamente invasivo, con raquianestesia, y alta hospitalaria sin sonda en 6 a 8 horas.

Como inconvenientes, el más importante es la falta de estadiaje quirúrgico, también el discomfort urinario que se provoca en un tercio de los pacientes, y por último el seguimiento con el PSA, que tiene un comportamiento en ocasiones caprichoso, y que hasta alcanzar el Nadir, en los primeros 5 años puede presentar picos que nos creen incertidumbre.

Por otro lado, al ser una técnica quirúrgica, se precisa un adiestramiento, y hoy por hoy en nuestro país habitualmente es necesario contar con un equipo multidisciplinar para su aplicación. Existe la posibilidad de obtener el título de operador-supervisor de isótopos radioactivos, para tener el urólogo la autonomía de realizar el implante con la ayuda de un físico.

\section{Indicaciones}

La indicación del tratamiento mediante braquiterapia siguiendo a las principales sociedades americanas y europeas, es en todos los pacientes con cáncer órgano confinado T1- T2, clasificados en tres grupos de riesgo, tomando como parámetros predictivos la dosificación de PSA y el grado de Gleason; bajo riesgo de recidiva de enfermedad, riesgo moderado o alto riesgo ${ }^{11,12}$.

\section{Contraindicaciones}

Como contraindicaciones a la técnica, tenemos las formales por problemas anestésicos, expectativa de vida menor de 5 años y enfermedad metastásica, y otras contraindicaciones relativas, que no es que sean inviables técnicamente, pero sí que tienen un alto índice de complicaciones como en la radioterapia pélvica o cirugía prostática previas.

En todos los pacientes realizamos una flujometría previa, y en aquellos que tienen un patrón obstructivo, bien por lóbulo medio, disectasia de cuello u obstrucción uretral, se les realiza una RTU, o uretrotomía previa, y en 12 semanas la braquiterapia.

En el caso de las próstatas mayores de 60cc se puede realizar el implante, siempre que ese crecimiento no esté asociado a un proceso obstructivo, teniendo la precaución de hacer una colocación de litotomía forzada para evitar el arco púbico. Todo esto sin obviar el tratamiento previo con bloqueo hormonal y la constatación previa al implante de la reducción de tamaño ${ }^{13}$.

Como complemento a este apartado de las contraindicaciones hacer una reflexión sobre un capítulo que a nuestro modo de ver es muy importante, y del que hay escaso reflejo en la literatura a pesar de su relevancia. Nos referimos a la patología rectal previa o concomitante. Es recomendable tener en cuenta la existencia de hernias inguinales recientes, cambios del ritmo intestinal que pueden ser signos satélites de patología tumoral a nivel del colon o recto. De igual manera deberemos prestar atención a los cuadros de cirrosis, hipertensión portal, síndrome próstato-hemorroidal, o patología hemorroidal simple. En estos casos es preferible la solución quirúrgica 12 semanas antes de la implantación de las semillas.

La patología que debemos descartar previamente a la braquiterapia es:

- Poliposis familiar

- Adenomas vellosos 
- Colitis ulcerosa

- Patologías asociadas al área rectal como: Hemorroides Internas o externas, criptitis, fisuras...

Como advertencia es necesario solucionar toda la patología rectal previa, y no se puede intervenir en la zona rectal, con limite en la bisagra recto-sigmoidea, hasta pasados 12 meses de la braquiterapia.

\section{Procedimiento del Implante}

Una vez documentado el paciente con PSA, ecografía, estudio anatomopatológico, flujometría, historial, y aceptado para implante se le realiza un estudio preoperatorio, y se solicitan las semillas

Establecido el tamaño prostático por ecografía, aplicando la formula del elipsoide, siguiendo el nomograma de $\mathrm{Wu}$ en función del tamaño prostático, se establece la actividad por semilla. Para las próstatas menores de 30cc sería entre 0,38 y $0,41 \mathrm{mCi}$, las comprendidas entre $30 \mathrm{y}$ 50 cc $(0,48-0,52 \mathrm{mCi})$ y en las que superan los 50cc $0,58 \mathrm{mCi}^{14}$.

Los implantes se pueden colocar por la técnica del preplanning, consistente en realizar dos semanas antes una simulación de situación de las semillas, y el día del implante tratar de reproducir la conformación previa en quirófano.

La otra sistemática que es la que nosotros utilizamos se realiza en tiempo real pudiendo hacer las correcciones en el propio acto quirúrgico. En principio parece que el intento de reproducir la misma posición anatómica según la técnica de preplanning, conlleva pequeños errores, que se evitan con la intraoperatoria. Asimismo con esta última se gana agilidad y tiempo. Hay pocos estudios comparativos que establezcan las ventajas o inconvenientes de cada una, aunque nosotros creemos desde la experiencia de haber utilizado las dos que esta última, cuando menos es más rápida, y permite hacer el ajuste de dosis en zonas infra o sobredosificadas ${ }^{13}$.

El predominio de implante periférico en nuestro centro, hace que el $70-80 \%$ de las semillas se depositen en la periferia, con una media de 12 a 15 agujas, y sólo 3 ó 4 a nivel central.

El tamaño no es inconveniente para el implante, siendo en nuestra casuística el 9\% de los pacientes con un volumen mayor de 50cc, y el 3\% superaban los 60cc. El mayor volumen implantado fue de 94cc, con 169 semillas, lo que nos sirvió de experiencia para no repetirlo en casos similares, ya que tuvo problemas de retención urinaria y sonda durante 6 semanas.

En posición de litotomía, bajo raquianestesia, en ocasiones complementada con sedación. Se realiza la asepsia del campo quirúrgico, rechazando la bolsa escrotal para exponer bien el periné. Se coloca una sonda vesical (Fooley $n^{\circ}$ 14) (Fig. 1).

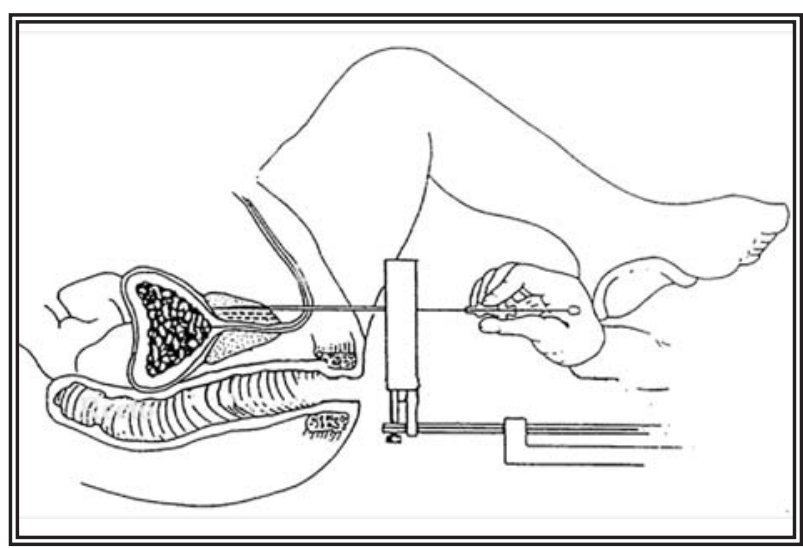

FIGURA 1

Se introduce el transductor rectal, colocado en el de cremallera (stepper de Accuseed) (Fig 2), que lo inmoviliza y permite un desplazamiento antero- posterior, y sobre el se coloca el template o rejilla graduada; se dan cortes ecográficos cada $5 \mathrm{~mm}$, desde el ápex a la base, identificando la uretra y el recto. Estos cortes se transfieren a un planificador con el que se calcula la distribución idónea de las semillas.

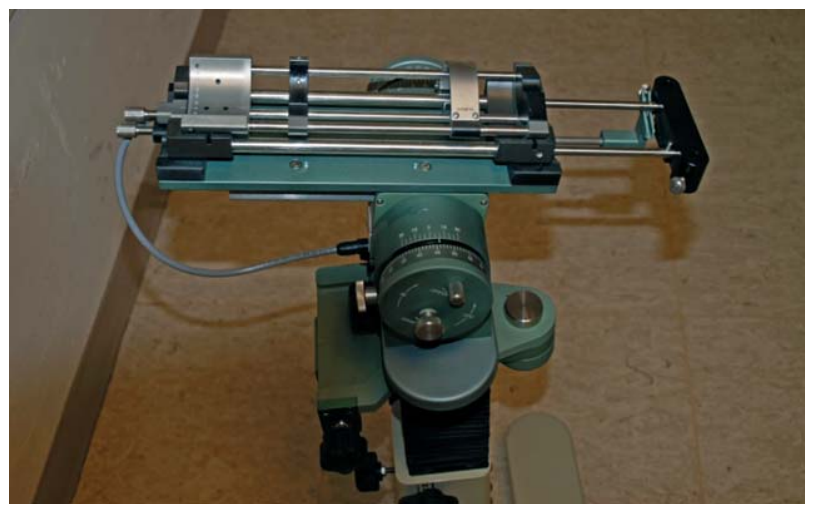

FIGURA 2 
El software utilizado para el cálculo de distribución de dosis proporcionada por las semillas es específico de Varian Medycal Systems, Variseed V7. $1^{15,16}$.

Los criterios dosimétricos actuales son de recibir el $98-100 \%$ al menos el 100\% de la dosis prescrita, $75 \%$ del volumen prostático el $150 \%$.

Con la situación del implante en el planificador, iniciamos la colocación de las agujas en el paciente, reajustando su situación en el software en tiempo real, y realizando una cistoscopia flexible para comprobar que no hay agujas ni en vejiga, ni en uretra.

Posteriormente se cargan las semillas en las agujas y se depositan en el tejido prostático. Nosotros utilizamos semillas en formato de Rapid-Strand, unidas por un hilo de material reabsorbible. Este sistema tiene como ventaja la disminución de probabilidad de migración de las semillas que con el método de implantes sueltos es más frecuente ${ }^{17}$ (Fig. 3).

Una vez son depositadas las semillas, éstas se localizan en su posición real y se calcula la distribución de dosis resultante basada en dicha posición. Esto permite hacer alguna modificación en el momento de completar el implante si fuera el caso ${ }^{18}$.

\section{Efectos de la radiación}

Los fotones producidos por la fuente radioactiva tienen una acción directa sobre el DNA, RNA, enzimas, proteínas y cualquier macromolécula celular provocando la muerte celular, e indirectamente tiene el mismo efecto al actuar las sustancias tóxicas, radicales, que provienen de la disociación de moléculas de agua al absorber la radiación ${ }^{19}$.

Las semillas implantadas con Iodo 125 permanecen con la máxima actividad radiactiva durante 60 días, disminuyendo progresivamente hasta desaparecer aproximadamente al año.

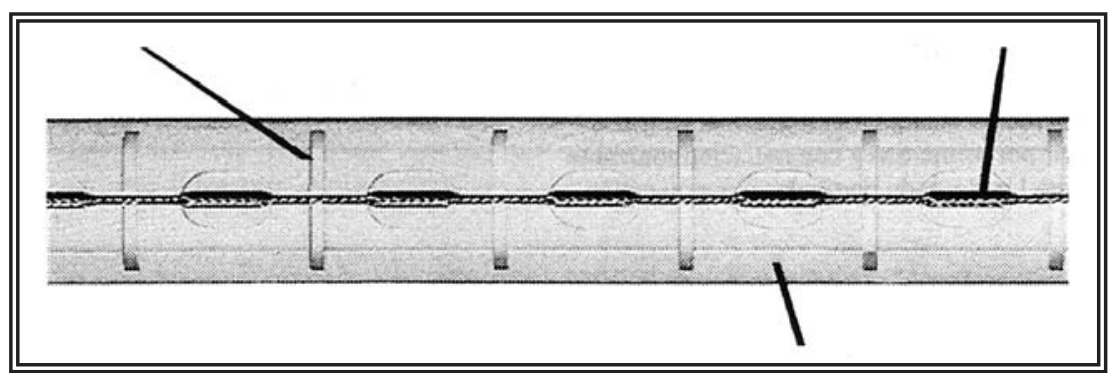

FIGURA 3. Semilla.

\section{Postimplante}

En nuestra casuística de más de 800 enfermos no ha habido complicaciones intraoperatorias ni en el postoperatorio inmediato ${ }^{32}$.

Una Radiografia simple de abdomen nos sirve para comprobar la correcta alineación de las semillas. La retirada de la sonda se realiza a las 4 horas, y a las 12 horas el paciente es dado de alta, prescribiéndole antiinflamatorios, alfa-bloqueantes, heparina de bajo peso molecular, y un antiséptico urinario (Fig. 4).

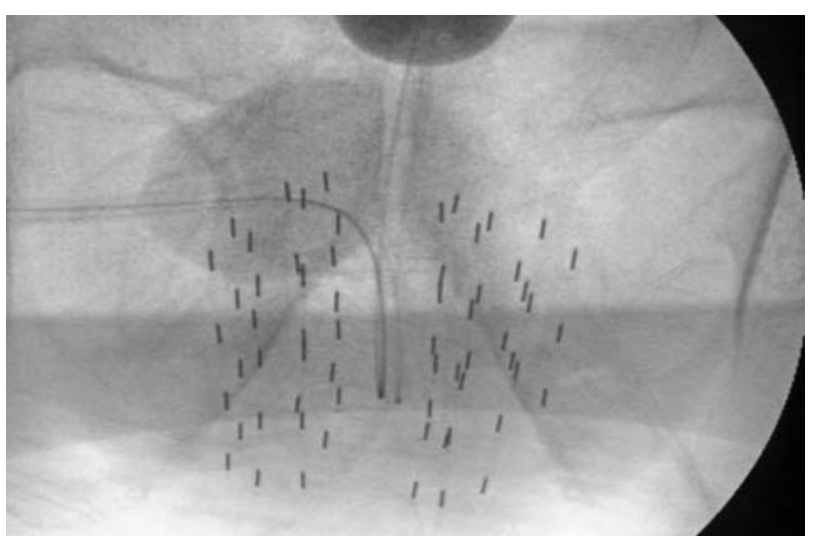

FIGURA 4

A los pacientes se les entrega un manual de precauciones y conducta a seguir a partir de la colocación del implante, aunque dada la escasa dosis de radiación del implante, los pacientes se pueden permitir llevar una relación social nor$\mathrm{mal}^{31}$.

El seguimiento de los pacientes es con una dosificación de PSA, cada tres meses los dos primeros años, y cada 6 meses los 5 años siguientes.

La recidiva biológica se considera según la ASTRO, cuando hay una elevación por encima del Nadir en tres mediciones consecutivas, o siguiendo el método de Phoenix, con Abramowitz cuando se eleva $2 \mathrm{ng} / \mathrm{ml}$ por encima del Nadir, este último parece que tiene más valor predictivo que el parámetro de la ASTRO $^{20,21}$.

\section{RESULTADOS}

De los 800 implantes de Iodo 125 realizados por nuestro grupo, 
vamos a describir las complicaciones más habituales que afectan al aparato urinario, al aparato digestivo y a la esfera sexual. La edad media de nuestra serie es de 68 años con rango de 49 a 83.

\section{Complicaciones urinarias}

Los sintomas urinarios de tipo irritativo son muy frecuentes, presentándolos hasta $2 / 3$ de los pacientes; generalmente son bien tolerados con tratamiento médico (alfa-bloqueantes y antiinflamatorios), su intensidad es leve-moderada en los primeros meses, mitigándose con el tiempo, destacando que al año desaparecen totalmente estos sintomas en la mayoría de los pacientes (Tabla 1).

Tabla 1. Tabla comparativa de incidencia de complicaciones urinarias

\begin{tabular}{lccccc}
\hline & \multicolumn{5}{c}{ Datos literatura médica } \\
& Stock & Wallner & Beyer & Blasko & $\begin{array}{c}\text { Experiencia } \\
\text { propia }\end{array}$ \\
\hline Retención & $4 \%$ & $14 \%$ & $4 \%$ & $7 \%$ & $3 \%$ \\
Incontinencia & $0 \%$ & $0 \%$ & $0,6 \%$ & $0 \%$ & $0,2 \%$ \\
\hline
\end{tabular}

En 14 pacientes realizamos RTU previa, por Lóbulo mediano (5), disectasia de cuello (4), RTU de próstata (5), y en 1 caso una Uretrotomía interna por estenosis uretral. El tiempo de espera para efectuar el implante fue de 12 semanas.

Esta previsión puede sin duda tener influencia en la escasa clínica obstructiva que presentaron nuestros pacientes, con la excepción de un caso en próstata de $94 \mathrm{cc}$, que obligó a sonda permanente durante 6 semanas.

En cuanto a la Incontinencia es mínima, con un $0,2 \%$ en nuestra serie ${ }^{22}$. Las lesiones rádicas que alteran la cicatrización del área del esfinter, pueden ser las que determinen los casos de incontinencia.

\section{Complicaciones rectales}

La complicación que con más frecuencia se presenta son los cuadros de rectitis, y alteraciones del ritmo intestinal con diarrea, que son de corta duración. Asimismo las rectorragias autolimitadas e intermitentes están presentes hasta en un $12 \%^{23-25}$
Pero la preocupación mayor en estos pacientes que presentan clínica rectal es evitar la progresión a fístula próstato-rectal, evitando maniobras (biopsias, cauterizaciones, o cirugía antes del año del implante), ya que estas manipulaciones pueden comprometer seriamente la vitalidad de estos tejidos bajo la radiación. La mejoría se consigue muy a menudo con lavativas de corticoides $^{26}$ (Tabla 2).

Tabla 2. Tabla comparativa de incidencia de patologia rectal

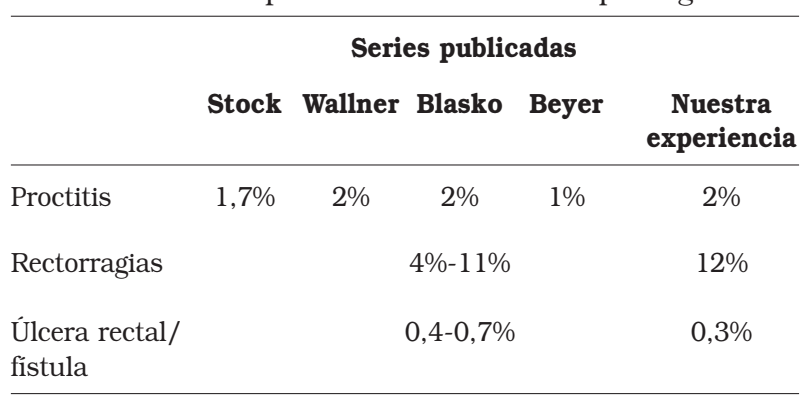

\section{Alteraciones sexuales}

La mayoría de los estudios, al igual que ocurre con la prostatectomía radical, recogen una incidencia variable de complicaciones, que van desde un 14 a un 50\%, influidas probablemente por el momento de recogida de los datos, ya que la impotencia se incrementa con el tiempo, tardando entre 1 y 5 años en manifestarse y confundiéndose en ocasiones los casos fisiológicos de envejecimiento con los secundarios a la braquiterapia. En nuestra serie el porcentaje es del $65 \%$ de media de preservación de la función sexual a lo largo de estos 7 años y medio (Fig. 5).



FIGURA 5. Mantenimiento función sexual en el tiempo. 
Trastornos menores de eyaculación, disminución o licuefacción del mismo, no son raros en el curso del primer año de tratamiento. Los tratamientos con inhibidores de la fosfodiesterasa son eficaces en el 80\% de los $\operatorname{casos}^{27,28}$.

De cualquier manera hay que destacar que es significativamente menor que con la Prostatectomía radical y con la radioterapia externa.

\section{Resultados biológicos}

El control biológico de la enfermedad lo realizamos con dosificación del PSA, la primera a los tres meses, siguiendo con análisis trimestral en los dos primeros años, y posteriormente semestral hasta el quinto año, y luego ya anual.

La cifra más baja del PSA (nadir), puede tardar en alcanzarse hasta dos años, siendo una cifra particular para cada individuo. Tampoco es anormal que se den pequeños repuntes en el PSA en los 5 primeros años, que no tienen en principio trascendencia ninguna, y que se asocian con procesos inflamatorios.

\section{DISCUSION}

La evolución del PSA en un grupo de 112 pacientes con un seguimiento de 7 años y medio se expresa la Fig. 6, donde se ve la desviación estándar de la media del PSA; coincide en esencia con las series publicadas, y aunque el seguimiento de 7 años y medio es amplio, dado que las recidivas en este tipo de cáncer son a largo plazo, se precisan mas estudios para confirmar la buena evolución de la supervivencia sin recidiva biológica, que es del $80 \%$, y como se expresa en la Fig. 7, con tasas del 93 y $83 \%$ respectivamente para los $\mathrm{T} 1$ y $\mathrm{T} 2$.

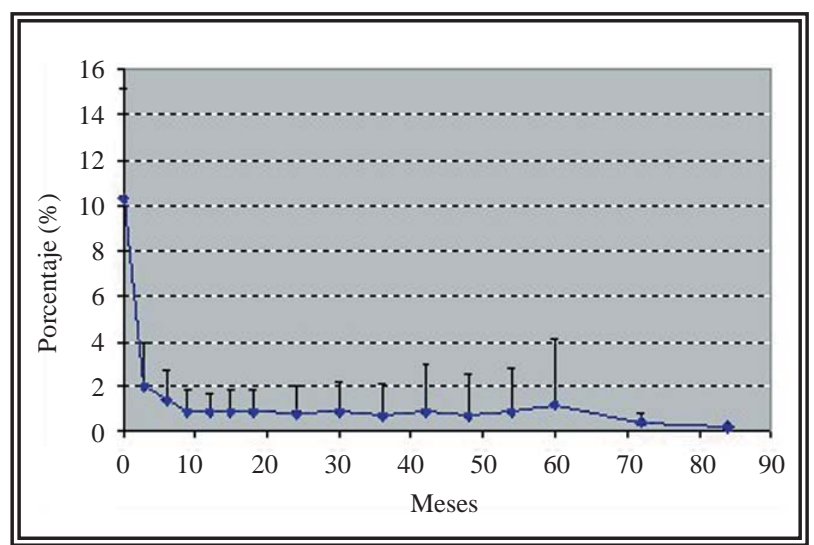

FIGURA 6. Evolución del PSA en baja tasa dosis (n=112).

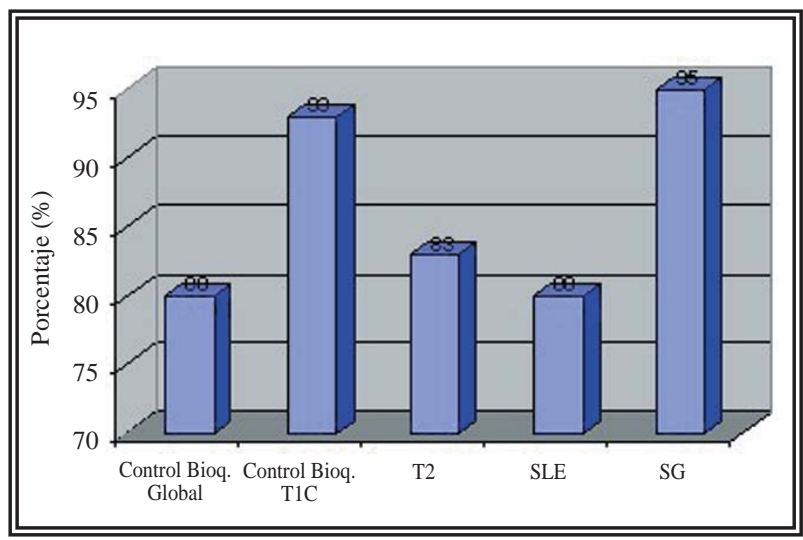

FIGURA 7. Resultados a 7,5 años.

Asimismo la supervivencia global es del 95\%. Hay que destacar, como se ve en la Figura 8, el mayor porcentaje de $\mathrm{T} 1$ un $70 \%$, frente al $27 \%$ deT2a, y sólo un 3\% de casos que rebasan el T2a.

La distribución en cuanto al Gleason, se ve en la Figura 9 con un marcado predominio del grupo $<$ de 7 , el 96\%, frente al 4\% > 7 .

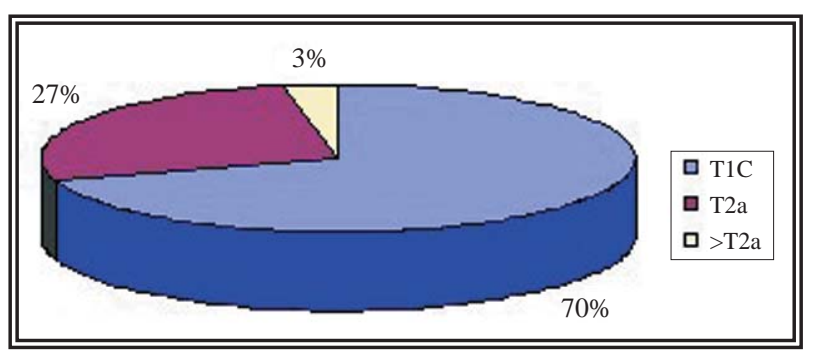

FIGURA 8. Distribución por estadios ( $n=112)$.

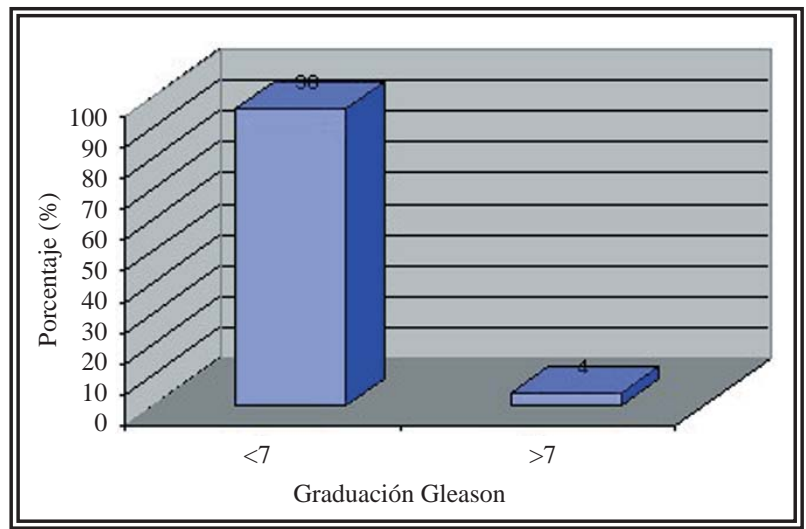

FIGURA 9. Distribución por Gleason.

La distribución del PSA por rangos demuestra (ver Fig. 10), cómo hay una selección marcada de los pacientes con un $64 \%$ de ellos con cifra inferior a $10 \mathrm{ng} / \mathrm{ml}$. 


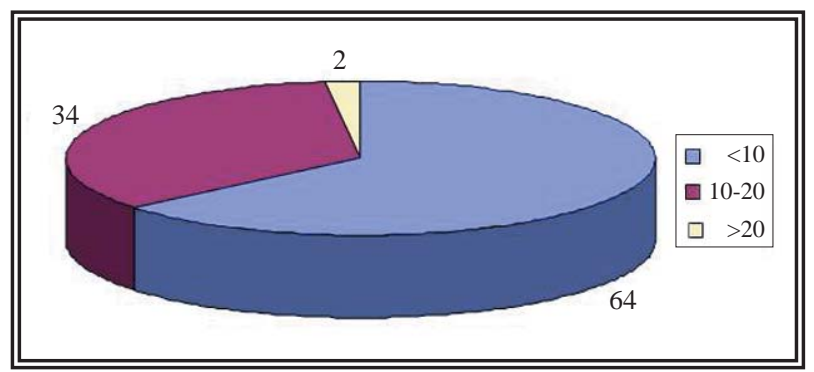

FIGURA 10. Distribución PSA (ng/ml) según rangos.

En cuanto a las complicaciones urinarias agudas post-implante de tipo obstructivo, en nuestra serie son muy escasas (3\%), en relación a otros autores $^{6,10,16,29}$, (Tabla 1) creemos que debido a las múltiples precauciones previas, flujometría, RTU previa de los lóbulos medianos, o de patología cervical obstructiva, y también el tipo de implante periférico, que produce menor inflamación aguda de la uretra. La conducta ante próstatas de tamaño mayor a los 60cc, es la universal de tratamiento con bloqueo androgénico, asociado o no a inhibidores de la 5 - $\alpha$-reductasa por un período mínimo previo al implante de 4 meses.

Con referencia a la incontinencia, autores como Stock, Blasko, Wallner6,15,22,30,33 no la incluyen entre sus complicaciones; en nuestra serie, aunque mínima ocupa un $0,2 \%$. Las lesiones rádicas que alteran la cicatrización del área del esfinter, pueden ser las que determinen los casos de incontinencia. (Tabla 1).

En relación a la morbilidad rectal que ocasiona el implante, como se muestra en la Tabla 2, las de tipo leve, como proctitis, rectorragias ocasionales, son bastante frecuentes, respondiendo bien a tratamientos locales con pomadas o enemas de corticoides ${ }^{42}$

La complicación más temida es la fístula rectal, que si bien en muchos casos, viene precedida de una manipulación previa del recto, antes de cumplirse el año del implante, en otras ocasiones sin antecedente alguno se presenta incluso doce meses después de la braquiterapia ${ }^{26,39,40,41,43}$. El problema es la difícil solución por la alteración tisular que compromete cualquier tipo de cirugía reparadora.

Nuestro grupo realiza desde hace algún tiempo una técnica de protección del recto, con inyección de ácido hialurónico en el espacio virtual próstato rectal, una vez puestas las agujas y antes de cargar las semillas radioactivas. La gran absorción de la radiación perirrectal, minimiza las complicaciones locales. Los resultados del tratamiento con inyección de Restylene ${ }^{\circledR}$ están aceptados para publicación en el International Journal of Radiation Oncology (Fig. 11).

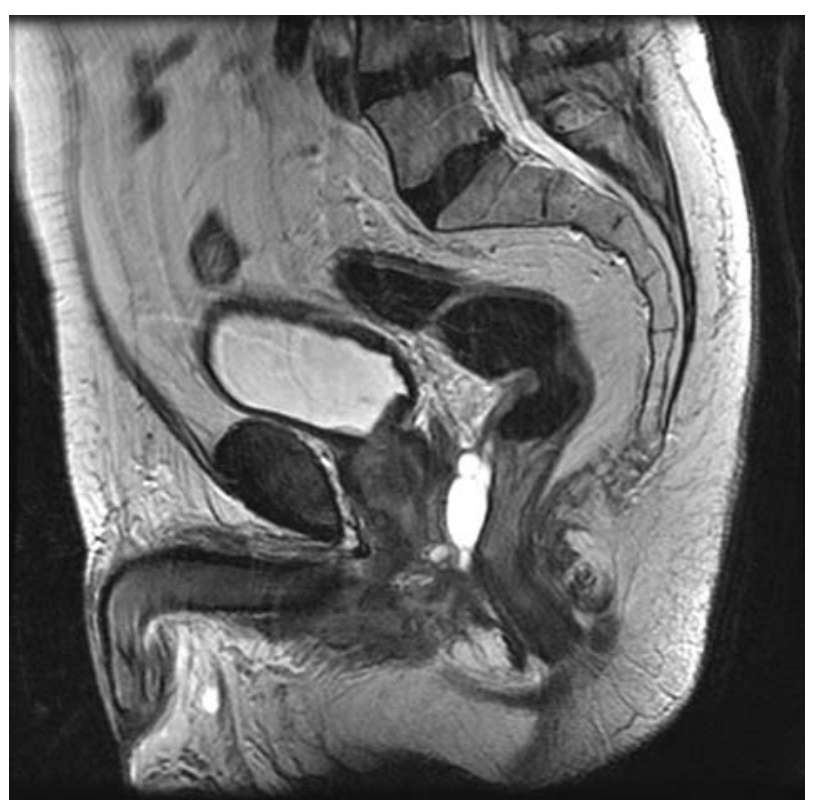

FIGURA 11. RNM de infiltración con Restylene ${ }^{\circledR}$.

\section{RNM de infiltración con Restylene ${ }^{\circledR}$}

En cuanto a la disfunción eréctil como se puede ver en la Fig. 5, nuestra experiencia es discordante con las otras series, y desde luego, vemos que así como las molestias urinarias mejoran con el tiempo y en su mayoría desaparecen en el plazo de un año, la disfunción eréctil empeora progresivamente en los 5 primeros años $31,35,42$.

\section{CONCLUSION}

Nuestra experiencia a lo largo de estos 7 años, con una casuística amplia, nos permite afirmar que con una buena selección de los pacientes candidatos, los resultados son satisfactorios, tanto en control biológico, como en supervivencia global. Asimismo la toxicidad de la braquiterapia, innegable como en cualquier tipo de radiación, se minimiza y alivia enormemente con precauciones que aunque parezcan evidentes no siempre se toman en cuenta; nos referimos al estudio funcional previo, a la no manipulación rectal en un plazo recomendable, y por último la utilización 
del gel de ácido hialurónico, entre otras medidas que son efectivas.

Asimismo la cistoscopia flexible para comprobar la correcta colocación de las agujas, antes del implante nos parece imprescindible.

La técnica está muy perfeccionada, y creemos que con la planificación en tiempo real, y el implante periférico en los estadios de bajo riesgo el resultado es equiparable al de la cirugía, aunque se precisan mas años de seguimiento para corroborar estas impresiones.

Aunque como decimos, la mejoría tecnológica es muy destacable, probablemente en el caso de la Baja Tasa haya tocado techo, y el relevo en el desarrollo y probablemente el futuro venga con los implantes temporales de Alta Tasa, que con los nuevos protocolos, los bajos costes de la fuente de energía de Iridio, se orientan a un tratamiento con cuatro dosis de Alta Tasa, obviando la complementaria externa.

\section{REFERENCIAS}

1. European Urology supplements. Volume 6 Issue 4 pag 405.- March 2007.

2. Pasteau O, Degrais P. The radium treatment of cáncer of the prostate. Arch Roentgen Ray. 1914;18:396.

3. Barringer BS. Radium in the treatment of carcinoma of the bladder and prostate. JAMA. 1917;68:1127.

4. Whitmore WF, Hilaris B, Grabstald H. Retropubic implantation of Iodine 125 in the treatment of prostatic cáncer. J Urol. 108(6):918-20.

5. Holm HH, Juul N, Pedersen JF, Hansen H, Strooyer I. Transperineal 125 Iodine implantation of the prostate for clinically localized prostate cáncer. J Urol. 1983;130(2): 283-286.

6. Blasko JC, Radge H, Grimm PD: Transperineal ultrasound guided implantation of the prostate : Morbidity and complications. Scand J Urol Nephrol Suppl. 1991;137:113118.

7. Fernández Madrigal F, Pérez Lacort L, González Tuero J, Junquera Villa JM. Tratamiento del cáncer localizado de próstata con implantes intersticiales de Au-198 radioactivo. Actas Urol Esp. 1986;10(2):127-130.

8. Pollack A, Zagars GK, Smith LG, Lee JJ, von Eschenbach AC, Antolak JA, Starkschall G, Rosen I. Preliminarfy results of a randomized radiotherapy dose-escalation study comparing 70 Gy with 78 Gy for prostate cáncer. J Clin Oncol. 2000;18(23):3904-3911.

9. Perez AA, Pilepich MV, Zivnuska F.. Tumor control in definitive irradiation of localized carcinoma of the prostate. Int J Radiat Oncol Biol Phys. 1986;12(4):523-531.

10. Hanks GE, Martz JH, Diamond IJ. The efect of dose on local control of prostate cáncer. Int J Radiat Oncol Biol Phys. 1988;15(6):1299-1305.

11. Ash D, Flynn A, Battermann J, de Reijke T, Lavagnini P, Blank L; ESTRO/EAU/EORTC recommendations on permanent seed implantation for localized prostate cancer. Radiother Oncol. 2000;57(3):315-321.
12. Nag S, Beyer D, Friedland J, Grimm P, Nath R. American Brachytherapy Society (ABS) recommendations for transperineal permanent brachyterapy of prostate Cancer. Int $\mathrm{J}$ Radiat Oncol Biol Phys. 1999;44(4):789-799.

13. Prada Gómez PJ, Juan Rijo G, Hevia Suárez M, Abascal García JM, Abascal García R. Técnica de planificación intraoperatoria en Braquiterapia prostática con implantes permanentes de I125. Arch Esp Urol. 2002 Dec;55(10): 1217-1223.

14. Wu A, Lee CC, Johnson M, Brown D, Benoit R, Miler R, Cohen J, Geis P, Chen AS, Kalnicki S. A new power law for determination of total 125I seed activity for ultrasoundguided prostate implants: clinical evaluations. Int J Radiat Oncol Biol Phys. 2000;47(5):1397-403

15. Wallner K, Roy J, Harrison L.: Dosimetry guidelines to minimize urethral and rectal morbidity following transperineal I125 prostate brachytherapy. Int $\mathrm{J}$ Radiat Oncol Biol Phys. 1995; 32: 465-471.

16. Simonian-Sauve M, Smart C, Mailleux H, Rosello R, Bladou F, Salem N. Iodine-125 transperineal prostate brachytherapy with preplanning technique: pre and post-implant dosimetry results analysis]. Cancer Radiother. 2003;7(2): 90-99.

17. Gao M, Wang JZ, Nag S, Gupta N. Seed migration effect in prostate brachytherapy. Med Phys. 2007 Feb;34(2):471480.

18. Nag S, Ciezki JP, Cormack R, Doggett S, DeWyngaert K, Edmundson GK, Stock RG, Stone NN, Yu Y, Zelefsky MJ; Clinical Research Committe, American Brachytherapy Society. Intraoperative planning and evaluation of permanent prostate brachytherapy: report of the American Brachytherapy Society. Int J Radiat Oncol Biol Phys. 2001 Dec;51(5): 1422-1430.

19. Latorre Travis E. Radiobiología Médica. ED. AC, Madrid 1979.

20. American Society for Therapeutic Radiology and Oncology Consensus Panel. Consensus statement: Guidelines for PSA following radiation Radiat Oncol Biol Phys. 1997;37: 31.

21. Abramowitz MC, Li T, Ross E et al. The nadir 2 definition of biochemical failure predicts for overall survival. Int $\mathrm{J}$ Radiat Oncol Biol Phys 2006;66: S207-2088.

22. Krumholtz JS, Michalski JM, Sundaram CP. Health-related quality of life and morbidity in patients receiving brachytherapy for clinically localized prostate cáncer. J Endourol. 2000;14(4):371-374.

23. Beyer DC, Priestley JB. Biochemical disease-free survival following I125 prostate implantation. Int $\mathrm{J}$ Radiat Oncol Biol Phys. 1997;37(3):559-563.

24. Wallner K, Roy J, Harrison L. Tumor control and morbidity following transperineal iodine 125 implantation for stage T1/ T2 prostatic carcinoma. J Clin Oncol. 1996;14 (2):449-453.

25. Gelblum DY, Potters L, Rectal complications associated with transperineal interstitial brachytherapy for prostate cancer. Int J Radiat Oncol Biol Phys. 2000;48(1):119-124.

26. Yurdakul G, De Reijke Tm, Blank Le, Rauws EA. Rectal squamous cell carcinoma 11 years after brachytherapy for carcinoma of the prostate. Urol. 2003;169(1):280.

27. Merrick GS, Butler WM, Lief JH, Stipetich RL, Abel LJ, Dorsey AT. Efficacy of sildenafil citrate in prostate brachytherapy patients with erectile dys-function. Urology. 1999;53(6):1112-1116.

28. Merrick GS, Butler WM, Dorsey At, Lief JH, Donzella JG. A comparison of radiation dose to the neurovascular bundles in men with and without prostate brachytherapyinduced erectile dysfunction. Int $J$ Radiat Oncol Biol Phys. 2000;48(4): 1069-1074. 
29. Beyer DC. Permanent prostate brachytherapy: the Arizona experience. Radiother Oncol. 2000;55:13-14.

30. Stock RG, Stone NN, DeWyngaert JK, Lavagnini P, Unger PD. Prostate specific antigen findings and biopsy results following interactive ultrasound guided transperineal brachytherapy for early stage prostate carcinoma. Cancer. 1996; 77:2386-2392.

31. Prada PJ, Hevia M, Juan G, Abascal JM, de la Rúa A, Abascal R et al. Braquiterapia de baja tasa en el cáncer de próstata localizado, resultados preliminares a 5 años. Arch Esp Urol. 2005;58(3):213-226.

32. Merrick GS, Wallner KE, Butler WM. Permanent interstitial brachytherapy for the management of carcinoma of the prostate gland. J Urol. 2003;169(5):1643-1652.

33. Wallner K, Lee H, Wasserman S, Dattoli M. Low risk of urinary incontinence following prostate brachytherapy in patients with a prior transurethral resection of the prostate. Int J Radiat Oncol Biol Phys. 1997 Feb;37(3):565569.

34. Blasko JC, Ragde H, Luse RW, Sylvester JE, Cavanagh W, Grimm PD. Should brachytherapy be considered a therapeutic option in localized prostate cancer?. Urol Clin North Am. 1996 Nov;23(4):633-650.

35. Stock RG, Stone MN, Tanuzzic. Sexual potency following interactive ultrasound-guided brachytherapy for prostate cáncer. Int $J$ Radiat Oncol Biol Phys. 1996;35(2):267-272.

36. Buron C, Le Vu B, Cosset JM, Pommier P, Peiffert D, Delannes $\mathrm{M}$ et al. Brachytherapy versus prostatectomy in localized prostate cancer: results of a French multicenter prospective medico-economic study. Int $\mathrm{J}$ Radiat Oncol Biol Phys. 2007 Mar;67(3):812-822.
37. Nag S, Ellis RJ, Merrick GS, Bahnson R, Wallner K, Stock R. American Brachytherapy Society recommendations for reporting morbidity after prostate brachytherapy. Int $\mathrm{J}$ Radiat Oncol Biol Phys. 2002;54(2):462-470.

38. Stone NN, Stock RG. Complications following permanent prostate brachytherapy. Eur Urol. 2002;41(4):427-33.

39. Cherr GS, Hall C, Pineau BC, Waters GS. Rectourethral fistula and massive rectal bleeding from iodine-125 prostate brachytherapy: a case report. Am Surg. 2001 Feb; 67(2):131-134.

40. Chrouser KL, Leibovich BC, Sweat SD, Larson DW, Davis $\mathrm{BJ}$, Tran NV et al. Urinary fistulas following external radiation or permanent brachytherapy for the treatment of prostate cancer. J Urol. 2005;173(6):1953-1957.

41. Prestidge BR, Bice WS. Urinary and rectal complications of comtemporary permanent transperineal brachyterapy for prostate carcinoma with or without external beam radiation therapy. Cancer. 2005;104(1):217-218.

42. Mallick S, Azzouzi R, Cormier L, Peiffert D, Mangin PH. Urinary morbidity after I125 brachytherapy of the prostate. BJU Int. 2003;92(6):555-558.

43. Theodorescu D, Gillenwater JY, Koutrouvelis PG. Prostatourethral-rectal fistula after prostate brachytherapy. Cancer. 2000;89(10):2085-91.

Correspondencia autor: Dr. J.M ${ }^{a}$ Abascal Junquera Servicio de Urologia. Hospital Universitario Central de Asturias Celestino Villamil, s/n - 33006 Oviedo (Asturias)

Tel.: 985108004

E-mail autor: josuvargas@hotmail.com

Información artículo: Original 